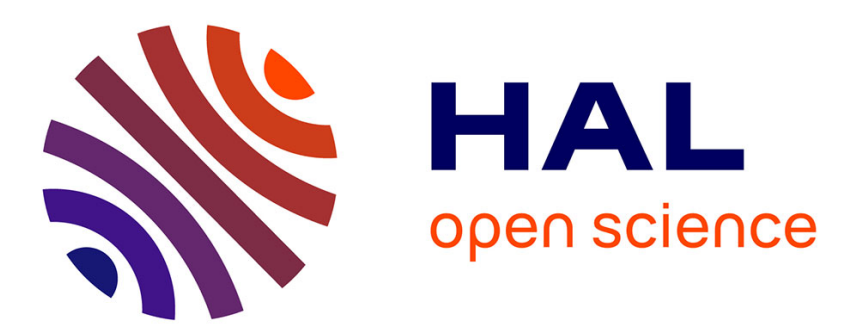

\title{
Chapitre 13. Informatisation de la " Drug Supply Chain " et sécurisation de la prise en charge du patient
}

\author{
Sarah Garidi, Julien Husson
}

\section{To cite this version:}

Sarah Garidi, Julien Husson. Chapitre 13. Informatisation de la "Drug Supply Chain " et sécurisation de la prise en charge du patient. Omar Bentahar; Smaïl Benzidia. Supply Chain Management de la santé, pp.233-249, 2019, 9782376871736. 10.3917/ems.bent.2019.01.0233 . hal-03092716

\section{HAL Id: hal-03092716 https://hal.science/hal-03092716}

Submitted on 27 Feb 2021

HAL is a multi-disciplinary open access archive for the deposit and dissemination of scientific research documents, whether they are published or not. The documents may come from teaching and research institutions in France or abroad, or from public or private research centers.
L'archive ouverte pluridisciplinaire HAL, est destinée au dépôt et à la diffusion de documents scientifiques de niveau recherche, publiés ou non, émanant des établissements d'enseignement et de recherche français ou étrangers, des laboratoires publics ou privés. 
CHAPITRE 13. INFORMATISATION DE LA « DRUG SUPPLY CHAIN »ET SÉCURISATION DE LA PRISE EN CHARGE DU PATIENT

\author{
Sarah Garidi, Julien Husson
}

in Omar Bentahar et al., Supply Chain Management de la santé

EMS Editions | «Gestion en liberté »

2019 | pages 233 à 249

ISBN 9782376871736

Article disponible en ligne à l'adresse :

https://www.cairn.info/supply-chain-management-de-la-sante---page-233.htm

Distribution électronique Cairn.info pour EMS Editions.

(C) EMS Editions. Tous droits réservés pour tous pays.

La reproduction ou représentation de cet article, notamment par photocopie, n'est autorisée que dans les limites des conditions générales d'utilisation du site ou, le cas échéant, des conditions générales de la licence souscrite par votre établissement. Toute autre reproduction ou représentation, en tout ou partie, sous quelque forme et de quelque manière que ce soit, est interdite sauf accord préalable et écrit de l'éditeur, en dehors des cas prévus par la législation en vigueur en France. Il est précisé que son stockage dans une base de données est également interdit. 


\title{
Chapitre 13. \\ Informatisation de la « Drug Supply Chain » et sécurisation de la prise en charge du patient
}

\author{
Sarah Garidi et Julien Husson
}

\section{Introduction}

L'environnement réglementaire difficile des établissements de santé (ES) les incite à investir dans des systèmes d'information afin de sécuriser la prise en charge médicamenteuse du patient. Les incertitudes liées à cette prise en charge poussent les ES à chercher des méthodes de flexibilité. C'est dans ce contexte incertain que les systèmes d'information (SI) sont utilisés pour diminuer la vulnérabilité de leur Supply Chain (SC) en gérant un flux d'informations de manière collaborative pour augmenter la visibilité (Basole et Bellamy, 2013).

Les SI apparaissent comme des outils au service de la SC en santé et de la sécurisation de la prise en charge du patient dont il est intéressant de vérifier la teneur sur un circuit comme celui de la Drug Supply Chain (DSC). La DSC également appelée la chaîne d'approvisionnement en médicament ou le circuit du médicament, selon Bedouch et al. (2009) englobe plusieurs étapes : «1/ la prescription médicale, 2/ la dispensation qui comprend l'analyse pharmaceutique et la délivrance des médicaments, 3/ l'administration des médicaments au patient par le personnel infirmier. Elle comprend : la gestion des commandes aux laboratoires, la gestion des stocks, des 
périmés, des retraits de lots et le choix des médicaments disponibles à l'hôpital ». Ce circuit est à fort risque pour les patients au regard de l'apparition des événements indésirables qui peuvent être multifactoriels et engendrer la rupture de la chaîne. La problématique que nous allons étudier dans cette recherche est la suivante : quel est l'apport de l'informatisation dans la prévention de la rupture de la DSC et dans la sécurisation de la prise en charge médicamenteuse du patient?

Pour cela, ce chapitre est structuré en quatre parties, une première consacrée au cadre théorique, aborde le SI comme un outil au service de la vulnérabilité de Supply Chain, puis établit le lien entre le système d'information et la sécurisation de la DSC. Une deuxième partie explique la démarche méthodologique. Ensuite, une troisième partie relate les résultats de la recherche en passant par l'apport de l'informatisation dans la sécurisation de la chaîne d'approvisionnement en médicament étudié. Enfin, une dernière partie est consacrée à la discussion des résultats et à la conclusion.

\section{Revue de la littérature}

\subsection{Le système d'information et la vulnérabilité de la Supply Chain (SC)}

Les SI sont définis par Reix et al. (2011) comme « un ensemble organisé de ressources qui permet d'acquérir ; traiter ; stocker, communiquer des informations dans les organisations ». Les SI utilisés dans la SC ont pour but de supporter et d'améliorer les processus de création, stockage, recherche, identification, transfert et intégration de connaissances (Alavi et Leidner, 2001). A ce titre, de nombreuses innovations en termes de logiciels de gestion et d'optimisation de la SC ont fait leur apparition pour répondre à ces défis (Said, 2006). Ces innovations complètent la panoplie des SI de la SC qui forment des «systèmes nerveux » plus flexibles, plus performants et capables de réagir en temps réel aux aléas de la SC (Ruel et Da Silva, 2016). Selon Lamarque (2014) les innovations qui suscitent de plus en plus d'intérêt sont « les nouveaux logiciels qui modélisent les flux et les stocks en intégrant les paramètres opérationnels d'une chaîne : cadence, délais, organisation des flux, règles de distribution, capacité et localisation des stocks, contraintes d'approvisionnement, niveau de service clients ». 
Le fonctionnement d'une SC se caractérise par des milliers d'événements et de changements d'état qui se déroulent chaque jour et qui constituent autant d'informations qui permettent aux organisations de prendre des décisions afin de s'adapter à l'évolution de leur environnement (Evrard-Samuel et al., 2011). Le rôle du SI est alors de simplifier au maximum la prise d'information pour analyser ces événements et permettre une prise de décision simplifiée au bon moment (Fabbe-Costes, 1997), mais malgré les avancées sur le plan des technologies de l'information, la vulnérabilité des SC, c'est-à-dire leur sensibilité aux événements, s'accroît (Peck, 2006).

Une vulnérabilité proportionnelle à l'augmentation des sources et des facteurs de risques qui provoquent des conséquences néfastes lorsqu'ils ne font pas l'objet d'une stratégie d'atténuation des risques (Jüttner et al., 2003). Dans le cas de la rupture de flux, Evrard-Samuel et Ruel (2016) expliquent que les SI « devraient être en mesure de fournir les informations nécessaires afin de rétablir et stabiliser les flux d'informations ", ils sont une clé de la réduction de la vulnérabilité dans la SC (Lavastre et al., 2014). En revanche, les auteurs constatent que la réalité dans les organisations est souvent différente, la capacité des SI à gérer ces ruptures de flux est négligée, augmentant ainsi la vulnérabilité de leur SC. Même si la plupart des organisations sont équipées de SI performants, les auteurs constatent que les problèmes de stocks, de délais, de ruptures d'approvisionnement demeurent présents et génèrent des coûts que les organisations cherchent à tout prix à éliminer.

\subsection{La DSC face à la rupture d'approvisionnement du patient en médicament}

L'hôpital actuel dans sa complexité est plus proche d'une immense chaîne logistique dont les différentes parties visent à satisfaire le patient que d'un système fonctionnant en vase clos. La comparaison avec une SC industrielle est alors évidente (Artiba et al., 2004). Cette complexité est due à la structure des processus logistiques et au nombre d'entités qui interagissent simultanément, de manière autonome ou concertée sur les flux (Chabrol et al., 2006).

La DSC n'échappe pas à cette complexité, également appelée la chaîne d'approvisionnement en médicament ou le circuit du médicament. La littérature de la Haute Autorité de Santé rapporte que ce circuit recouvre en pratique deux chaînes : 
- La chaîne « logistique » (ou flux physique) qui relie respectivement les fabricants, les centrales d'achat, la pharmacie à usage interne, les services cliniques et enfin le patient pour l'administration du médicament.

- La chaîne « clinique » qui voit se succéder la prescription, la dispensation et enfin l'administration au patient.

Cette définition est complétée par l'agence Nationale d'Appui à la Performance (ANAP) par le « flux d'information » qui permet l'identification du patient et la traçabilité de l'information tout au long de l'acheminement du médicament vers le patient.

Les publications de la HAS mettent en avant la règle des $5 \mathbf{B}$ comme fil conducteur de la sécurisation de la DSC. Son objectif est que le Bon patient reçoive le Bon médicament au Bon moment, à la Bonne dose, selon la Bonne voie d'administration.

Cependant, pour atteindre cet objectif, plusieurs prérequis sont indispensables selon la littérature :

- la prescription du médicament adapté à la pathologie du patient ;

- l'identification du patient au moment de la prescription et de l'administration ;

- la disponibilité du bon médicament au lit du patient, à la bonne dose et la bonne forme ;

- le respect de la bonne voie d'administration ;

- la gestion des dysfonctionnements : les repérer, les recenser, les analyser et les corriger si possible.

Un ensemble de prérequis qui conditionne l'usage sécurisé des médicaments pour la prise en charge du patient. A défaut, on parle alors d'iatrogénie médicamenteuse, lorsque « la thérapeutique médicamenteuse induit des effets, réactions, événements ou accidents indésirables, tant en raison des effets propres des médicaments concernés qu'à cause du contexte et des modalités de leur utilisation » (Bedouch et al., 2009). La iatrogénie peut provenir d'un effet indésirable ou d'une erreur médicamenteuse définie comme tout événement évitable résultant d'un dysfonctionnement non intentionnel dans l'organisation de la prise en charge médicamenteuse du patient (Bates et al., 1995).

La littérature révèle que des études réalisées en France et à l'étranger laissent supposer qu'un séjour hospitalier sur dix est marqué par un événement indésirable médicamenteux dont le tiers est qualifié de grave (Bedouch et al., 2009). Dans une étude, Bernheim et al. (2005) 
indiquent que quasiment une erreur sur deux, commise au niveau du circuit du médicament, atteindra le patient. Par ailleurs, Ellenberg et De Paris (2003) précisent qu'un événement indésirable est rarement le résultat d'une seule erreur, mais bien souvent d'une série d'erreurs ou d'événements produits par de nombreux facteurs organisationnels, professionnels et personnels.

A ce sujet, l'informatisation du circuit du médicament hospitalier a fait l'objet de nombreuses publications qui mettent en avant ses avantages sur l'incidence des erreurs médicamenteuses (Bates et al., 1995 ; King et al., 2003 ; Koppel et al., 2005 ; Vialle et al., 2011).

\section{Méthodologie}

La revue de littérature permet de comprendre que la DSC gère à la fois des flux physiques, cliniques ainsi que des flux d'informations. Dans ce travail, l'approche qualitative de nature exploratoire permet de s'interroger sur l'impact d'un événement indésirable sur la rupture de la chaîne d'approvisionnement en médicament nécessaire à la prise en charge du patient et sur le rôle de l'informatisation dans la prévention et la gestion de cette rupture.

Selon Yin (2013), « utiliser une étude de cas unique, présentant toutes les conditions nécessaires pour tester une théorie, peut confirmer, challenger ou enrichir la théorie ». C'est le choix qui a été fait pour ce travail de recherche qui porte sur un seul établissement de santé. Nous proposons d'étudier le cas de la DSC du CSSR, un établissement de santé d'une capacité de 90 lits et places, dont le circuit du médicament est informatisé depuis 2004. L'établissement dispose d'un dossier patient informatisé (DPI) OSIRIS accessible à une équipe pluridisciplinaire formée à son utilisation. Le circuit du médicament est géré par un pharmacien responsable du système de management de la qualité de la prise en charge médicamenteuse. Celui-ci est président du COMEDIMS (Commission du Médicament et des Dispositifs Médicaux Stériles), instance chargée de garantir le bon fonctionnement du circuit du médicament dans l'établissement. La pharmacie à usage interne (PUI) de l'établissement permet l'approvisionnement en médicament de deux unités de soins (hospitalisation de jour et hospitalisation complète) dont la gestion des stocks est assurée via le logiciel OSIPHARM. Les prescriptions informatisées des médicaments sont réalisées par les médecins. Elles sont analysées par le pharmacien et validées avant d'être dispensées no- 
minativement aux deux unités. L'administration des médicaments au patient est réalisée par l'infirmière et la traçabilité est informatisée. Comme dans tout établissement de santé, la prise en charge médicamenteuse du patient donne lieu à un suivi régulier par les infirmières, sous la responsabilité du médecin référent. Dans ce sens, l'évolution de la pathologie du patient peut induire des modifications de prescriptions que l'infirmière doit prendre en compte pour effectuer une commande de médicaments à la pharmacie. Le dysfonctionnement de cette étape entraîne la rupture de la chaîne d'approvisionnement du patient en médicaments. La vulnérabilité de la DSC à cette étape est un sujet que l'on souhaite explorer dans ce chapitre afin de comprendre l'apport de l'informatisation face à la vulnérabilité de la DSC dans la sécurisation de la prise en charge du patient.

Dans cette étude, la collecte des données a été réalisée suivant deux techniques selon le modèle proposé par Yin (2013) : des entretiens semi-directifs (18 entretiens) pour comparer et compléter les points de vue des professionnels en lien direct avec le circuit étudié (tableaux 1 et 2) et des observations effectuées au CSSR. Les entretiens semi-directifs ont l'avantage de laisser une flexibilité qui permet de mettre en lumière toutes les idées et les connaissances des interviewés. Ils ont été enregistrés puis retranscrits à des fins d'analyse.

Nous avons complété cette collecte par une exploration documentaire portant sur les déclarations des événements indésirables issus du logiciel de gestion des risques de l'établissement. Par ailleurs, les observations effectuées dans les services, nous ont permis de saisir l'atmosphère de travail et de générer des conclusions en rapport avec les données collectées lors des entretiens. Ce choix méthodologique avait pour but de limiter le degré de subjectivité analytique de notre recherche portant sur une activité très complexe.

Tableau 1. Personnes interrogées externes au CSSR

\begin{tabular}{llc}
\hline \multicolumn{1}{c}{ Interviewés } & Durée (min) & \multicolumn{1}{c}{ Date } \\
\hline $\begin{array}{l}\text { Direction de la qualité et des relations avec les } \\
\text { personnes accompagnées }\end{array}$ & $20 ; 30$ & $\begin{array}{l}20 / 12 / 2017, \\
11 / 10 / 2017\end{array}$ \\
\hline Formateur - Expert Dossier pharmacie & 40 & $07 / 11 / 2017$ \\
\hline Formateur au Dossier patient informatisé DPI & 35 & $08 / 11 / 2017$ \\
\hline Responsable qualité et gestion des risques - CSSR autre & 30 & $11 / 10 / 2017$ \\
\hline
\end{tabular}


Tableau 2. Personnes interrogées internes au CSSR

\begin{tabular}{lll}
\hline \multicolumn{1}{c}{ Interviewés } & \multicolumn{1}{c}{$\begin{array}{c}\text { Durée } \\
(\mathrm{min})\end{array}$} & \multicolumn{1}{c}{ Date } \\
\hline $\begin{array}{l}\text { Cadre de santé - coordinateur de la gestion des } \\
\text { risques associés aux soins }\end{array}$ & $\begin{array}{l}20,50,15, \\
15,60,30\end{array}$ & $\begin{array}{l}26 / 07 / 2017,24 / 07 / 2017, \\
21 / 07 / 2017,30 / 10 / 2017, \\
16 / 11 / 2017,19 / 01 / 2018\end{array}$ \\
\hline $\begin{array}{l}\text { Pharmacien - Responsable du management } \\
\text { de la qualité de la prise en charge médicamen- }\end{array}$ & $20,50,15$, & $26 / 07 / 2017,24 / 07 / 2017$, \\
teuse & $60,30,45$ & $19 / 01 / 2017,16 / 11 / 2017$, \\
\hline Infirmière - IDE A & 30 & $03 / 08 / 2017$ \\
\hline Infirmière - IDE B & 20 & $10 / 08 / 2017$ \\
\hline Infirmière - IDE C & 30 & $10 / 08 / 2017$ \\
\hline Infirmière - IDE D & 30 & $14 / 08 / 2017$ \\
\hline Infirmière - IDE E & 20 & $08 / 08 / 2017$ \\
\hline Infirmière - IDE F & 30 & $08 / 08 / 2017$ \\
\hline Infirmière - IDE G & 20 & $03 / 08 / 2017$ \\
\hline Infirmière - IDE H & 40 & $08 / 08 / 2017$ \\
\hline Médecin I & 30 & $16 / 11 / 2018$ \\
\hline Médecin II & 15 & $26 / 07 / 2017$ \\
\hline Administrateur réseau & 60 & $26 / 07 / 2017$ \\
\hline Directeur & 20 & $16 / 11 / 2017$ \\
\hline Légende : IDE : Intmin & & \\
\hline
\end{tabular}

Légende : IDE : Infirmière diplômée d'état ; CSSR : Centre de Soins de Suite et de Réadaptation ; DPI : Dossier Patient Informatisé.

\section{Résultats}

\subsection{Modélisation et description de la DSC étudiée}

La modélisation de la Drug Supply Chain étudiée permet d'avoir une première vision globale de ses différents flux (clinique, logistique, information). La figure 1 présente la cartographie des différentes étapes multidisciplinaires, interdépendantes et nécessaires à l'approvisionnement du patient en médicament. Elle reflète l'alignement des différents intervenants de cette chaîne de façon à satisfaire le client final qui est le patient. Aussi, elle présente les différentes perturbations de la DSC qui peuvent induire une rupture d'approvisionnement. 
Cette cartographie est une analyse dynamique qui a pour but d'apporter de la transparence dans la présentation de la complexité de la DSC étudiée. L'approche itérative adoptée lors de sa réalisation a nécessité le choix d'un langage de modélisation compréhensible par les acteurs interrogés et respectant un niveau de granularité adaptée à notre étude. Cette approche a consisté à soumettre la cartographie à l'avis des différents intervenants interrogés pour qu'elle soit le reflet de leur pratique au quotidien.

Afin de comprendre le rôle de l'informatisation dans la sécurisation de la DSC étudiée, une description succinte de son organisation paraît nécessaire. L'objectif est de faire le parallèle entre les différents points sensibles identifiés par les professionnels interrogés, susceptibles de provoquer une rupture de la chaîne d'approvisionnement en médicament et les solutions offertes par les SI pour les sécuriser.

La première étape du circuit est la prescription médicamenteuse informatisée. Les médecins sont invités à privilégier une prescription utilisant le livret thérapeutique paramétré dans le logiciel afin de garantir la disponibilité du médicament dans les stocks de la PUI ou à défaut, une mise à disposition rapide.

Ensuite, l'ensemble des prescriptions sont analysées par le pharmacien et dispensées après avoir été validées. Elles peuvent aussi faire l'objet d'une substitution auquel cas, un commentaire est tracé dans le logiciel par le pharmacien.

Une fois l'étape validée, les unités de soins bénéficient ainsi d'une dispensation nominative. Pour cela, le pharmacien prépare les médicaments prescrits pour les patients dans des sacs nominatifs qui sont placés dans une caisse pour être transportés dans les services de soins. Chaque sachet contient les médicaments nécessaires pour 5 jours de traitement. Les sachets sont rangés dans un tiroir dédié au patient dans le chariot à médicaments.

La prise en charge du patient nécessite parfois des changements ou des renouvellements de prescriptions auxquelles les infirmières doivent rester vigilantes. Celles-ci doivent enclencher systématiquement une commande à la pharmacie pour éviter une rupture de la DSC. Les médicaments et la quantité à commander sont obtenus après vérification de la prescription et des stocks restants dans le chariot à médicaments.

Le support utilisé pour la commande est un cahier qui assure la liaison entre les services de soins et le pharmacien. Pour chaque commande, l'infirmière doit retranscrire sur le cahier : le nom du 


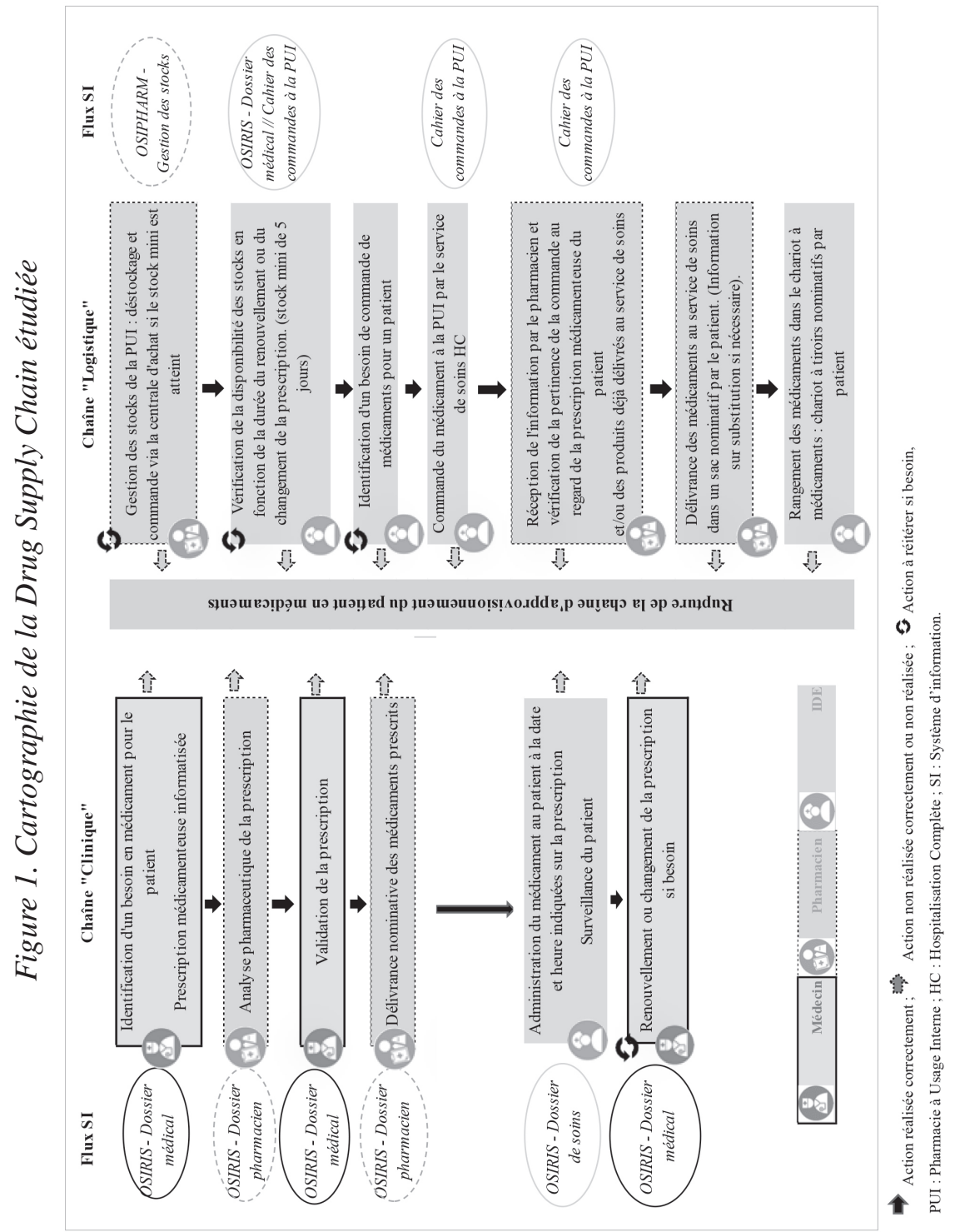


patient, la liste des médicaments à commander, la quantité, le dosage. Cette retranscription a été identifiée par les professionnels interrogés comme une pratique fortement à risque et source d'erreurs.

Lors de l'analyse des différents entretiens réalisés, il apparaît que la procédure de réalisation des commandes n'est pas uniformisée entre les différentes infirmières chargées de réaliser cette action.

Ainsi, une partie des infirmières procèdent à la vérification des tiroirs nominatifs du chariot des médicaments, tiroir par tiroir, contrairement à une autre partie qui privilégie la vérification par ordre, de la chambre $n^{\circ} 1$ à la chambre $n^{\circ} 31$. Les deux parties vérifient la disponibilité du traitement pour le patient pour une durée de 5 jours en fonction de la prescription médicale.

«Pour chaque tiroir ; je vérifie le nom du patient, je prends la feuille du traitement et la prescription sur OSIRIS. Pour chaque produit, je compte le reste dans le tiroir et prévois la consommation pour le nombre de jour prévu, soit 5 jours. » IDE B

«La commande se fait, patient par patient de la chambre 1 à la chambre 31. Je vérifie la quantité de chaque traitement et si les traitements correspondent à la prescription médicale. » IDE C

Aussi, une divergence apparait sur le type de support utilisé pour vérifier la prescription. Une large majorité des IDE(s) utilise l'impression de la prescription. Les impressions des prescriptions médicamenteuses sont rangées dans un classeur, mais ne sont pas systématiquement mises à jour, au risque de ne pas prendre en compte des changements de dernière minute réalisés par les médecins dans le DPI. Seulement 3 infirmières sur 8 ont déclaré qu'elles utilisent directement le logiciel OSIRIS pour vérifier la prescription médicale et les besoins éventuels du patient.

"Les impressions papier sont plus faciles à suivre ligne par ligne avec une feuille ou une règle. Le suivi sur OSIRIS est compliqué et provoque une fatigue visuelle. » IDE E

La raison évoquée pour justifier le manque d'utilisation de la prescription informatisée est un défaut d'ergonomie de l'outil, entrainant une lisibilité difficile et contrainte par l'affichage réduit des lignes de prescription et des commentaires importants saisis par les médecins pour certains médicaments.

Malgré ces points, le souhait d'informatiser la commande a été communiqué à plusieurs reprises et cela afin d'uniformiser la procédure de réalisation. L'informatisation est vue comme un gain de temps considérable permettant aussi de sécuriser cette étape en évi- 
tant : des erreurs de retranscription, des erreurs de patients, des erreurs de traitements et aussi des oublis de commandes.

« Je mets 2 heures environ pour réaliser la commande, c'est trop long de réécrire chaque patient puis chaque traitement sur le cahier. Ça serait mieux de cliquer sur OSIRIS et d'avoir le traitement à commander pour plus de facilité. » IDE G

«J'utilise la prescription médicamenteuse sur OSIRIS, c'est pour cela qu'il serait mieux d'avoir la commande en parallèle de la prescription. » IDE F

Enfin, l'analyse des entretiens a mis en évidence une utilisation non optimisée du logiciel et des différentes solutions offertes par celui-ci. La raison avancée est souvent un manque d'information ou de formation sur le logiciel. Les professionnels savent que les établissements de santé sont fortement impactés par le virage numérique et l'informatisation et ne souhaitent pas rater cette transition.

«Pas évident quand on ne connaît pas le logiciel car il y a des subtilités pas toujours faciles à repérer. » IDE B

«Commande à réaliser sur ordinateur avec sur une partie de l'écran, la prescription Osiris et sur l'autre partie la commande, un clic sur le nom du patient permettant d'afficher la commande des traitements qui lui sont propres. » IDE H

\subsection{Analyse des événements indésirables induisant la rupture de la DSC}

Cette partie présente le résultat de l'analyse des événements indésirables déclarés sur l'année 2017 ; une base de 143 événements indésirables (EI) a été analysée. Trente EI (21\%) sont liés à la DSC, parmi lesquels il apparaît que les événements liés à des problèmes de commandes de médicaments par les services de soins sont les plus fréquents ; ils représentent $63 \%$. Ces événements provoquent une rupture de la chaîne d'approvisionnement en médicaments et perturbent la prise en charge du patient. Ils sont parfois dangereux lorsque la stabilité du profil pathologique dépend du médicament en question et non administré à temps. 
Tableau 3. Analyse des événements indésirables déclarés

\begin{tabular}{lccc}
\cline { 2 - 4 } & $\begin{array}{c}\text { Nombre } \\
\text { d'EI }\end{array}$ & $\begin{array}{c}\text { Pourcentage } \\
\text { par EI }\end{array}$ & $\begin{array}{c}\text { Pourcentage } \\
\text { par EI liés à } \\
\text { la DSC }\end{array}$ \\
\hline $\begin{array}{l}\text { Nombre d'événements indésirables } \\
\text { (EI) analysés }\end{array}$ & 143 & & \\
\hline $\begin{array}{l}\text { Nombre d'EI liés à la Drug Supply } \\
\text { Chain }\end{array}$ & 30 & $21 \%$ & \\
\hline $\begin{array}{l}\text { Nombre d'EI liés à des problèmes } \\
\text { de commandes de médicaments }\end{array}$ & 19 & $13 \%$ & $63 \%$ \\
\hline
\end{tabular}

Figure 2. Répartition des EI liés à la DSC dont les EI liés aux problèmes de commandes

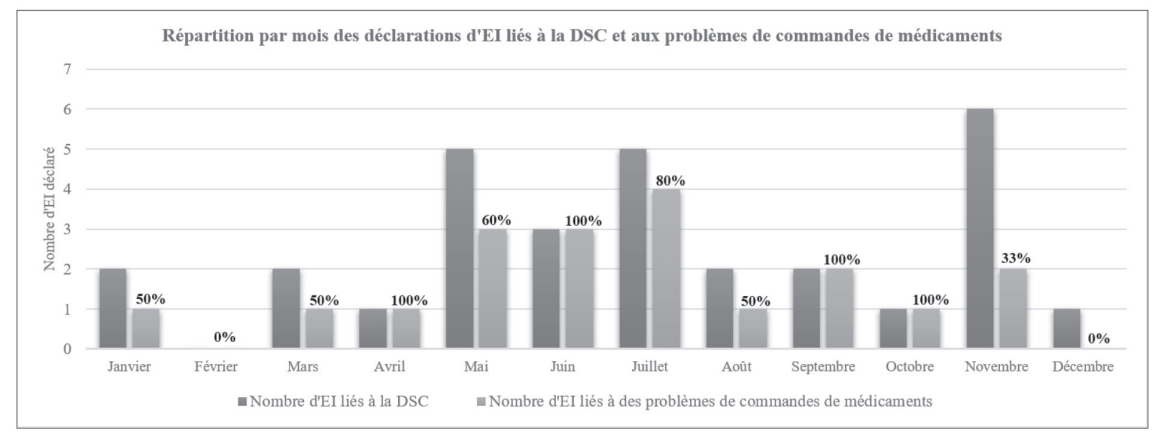

L'analyse des événements liés à des problèmes de commandes de médicaments, en plus de perturber la prise en charge médicamenteuse du patient, a mis en avant d'autres dysfonctionnements :

- un défaut de formation ou un manque d'information des IDE sur la substitution des médicaments ;

- les prescriptions hors livret thérapeutique perturbent fortement la chaîne d'approvisionnement en médicaments ;

- le support de commande est inadapté à une traçabilité optimale et une sécurisation du circuit (risque lié à la retranscription sur le cahier des commandes) ;

- la méconnaissance des procédures élaborées par le COMEDIMS pour sécuriser le circuit et l'absence de procédures pour la réalisation des commandes ; 
- la lisibilité et l'ergonomie du support de prescription à améliorer ;

- le manque d'anticipation des besoins en médicaments lors des week-ends et des jours fériés.

\subsection{Les solutions offertes par le système d'information pour sécu- riser la DSC}

Comme indiqué précédemment, le CSSR a intégralement informatisé son circuit du médicament avec le logiciel OSIRIS sur la partie dossier patient informatisé et le logiciel OSIPHARM sur la partie gestion des stocks de la PUI. Deux logiciels dont l'interface est opérationnelle.

Le DPI offre la possibilité de paramétrer des alertes pour les patients pour lesquels le médecin doit renouveler le traitement de fond, revoir un traitement ponctuel ou réévaluer un traitement. Ces alertes utilisées par les médecins permettent une continuité dans le traitement médicamenteux du patient.

Par ailleurs, le logiciel dispose d'un module « commande à la pharmacie » qui offre la possibilité de faire une commande de médicaments globale ou nominative. La première, regroupe la consommation en médicaments de plusieurs patients hospitalisés dans un service, la deuxième est quant à elle personnalisée et individuelle et ne prend en compte que le traitement d'un seul patient. De plus, ce module offre une visibilité à l'infirmière sur la disponibilité des stocks du médicament à la pharmacie. En revanche, ces deux modes de commandes ne sont actuellement pas utilisés par les services de soins du CSSR.

La validation d'une commande par l'IDE à l'aide de ce module envoie une alerte à la pharmacienne pour qu'elle puisse gérer la commande et lui donner une suite. La transmission de l'information entre les services de soins et la pharmacie est ainsi totalement dématérialisée et sécurisée par le logiciel.

Dans ce sens, les professionnels concernés par cette étape du circuit du médicament mettent en avant les avantages de l'informatisation dans la réduction du risque lié à la retranscription faite sur le cahier des commandes et du risque d'oubli de commande entrainant une rupture de la DSC. 


\section{Discussion}

La description des différentes étapes de la Drug Supply Chain laisse apparaître que l'informatisation offre des solutions qui permettent une certaine maîtrise et une sécurisation de la gestion de l'approvisionnement en médicaments. Cela rejoint le constat de Livolsi et Fabbe-Costes (2004) qui indique que le couplage entre les flux physiques et les flux informationnels est indispensable au pilotage de la SC. Dans notre cas, ce couplage doit toutefois se conformer aux réglementations applicables au circuit du médicament.

Par ailleurs, l'analyse des déclarations des événements indésirables a mis en exergue que la DSC étudiée est fortement vulnérable aux problèmes de commandes entre le service de soins et la pharmacie. Cette vulnérabilité est causée par une perturbation ou une rupture du flux informationnel conduisant à une rupture du flux physique.

Ces problèmes peuvent être résolus par l'utilisation de la commande informatisée offerte par le logiciel de gestion du dossier patient. Ainsi, le système d'information permettra à la fois de prévenir les ruptures d'approvisionnement en médicaments, pallier les ruptures de flux et maintenir la continuité de la prise en charge médicamenteuse. A ce stade Ponomarov et Holcomb (2009) parlent de la résilience de la SC. Evrard Samuel et Ruel (2013) expliquent qu' « en cas de rupture brutale des flux, le SI devrait être en mesure de fournir les informations nécessaires afin de rétablir et stabiliser les flux d'informations ». Dans notre cas, l'informatisation du processus de commande va permettre d'adapter la DSC à prévenir les ruptures d'approvisionnement en médicaments et aura pour but également d'améliorer la réactivité de la DSC face à un événement imprévu.

En revanche, il est important de souligner qu'un manque de connaissances de l'outil informatique entraine à la fois une perte de temps, mais aussi un éventuel déficit d'adhésion au système provoquant ainsi une vulnérabilité de la SC (Vialle et al., 2011). Conjointement à cela, notre étude a permis également de vérifier que le recours à des messages d'alerte est bénéfique dans certaines situations car il permet de prévenir la rupture de la DSC, mais il est important d'indiquer aussi qu'il peut s'avérer contre-productif : un excès de messages d'alerte aboutit le plus souvent à une désactivation ou à un défaut de lecture (Bouchand, 2007 ; Koppel et al., 2005).

Ce qui nous amène à aborder l'importance du facteur humain dans la sécurisation de la DSC par l'informatisation. Comme l'indiquent 
Koppel et al. (2005), il est indispensable d'accompagner le déploiement de l'informatisation en se concentrant sur les facteurs humains et organisationnels (Schmitt D. et al., 2001 ; Bourget et al., 2007). Le renforcement de la vigilance du personnel par la formation et le suivi des compétences par une grille d'habilitation contribuent aussi à l'amélioration de la qualité du processus (Ducret, 2016).

\section{Conclusion}

Notre étude s'intéresse à l'informatisation de la Drug Supply Chain et son rôle dans la prévention des ruptures de l'approvisionnement du patient en médicaments et dans la réduction de la iatrogénie médicamenteuse induite. Les recherches académiques en lien avec notre sujet notamment en sciences de gestion, restent insuffisantes au regard des facteurs influençant la sécurisation de ce circuit (organisationnels, techniques, humains, stratégiques et réglementaires, etc.).

Le cas étudié montre que l'informatisation permet de contribuer fortement à la sécurisation de la prise en charge médicamenteuse du patient. Elle a l'avantage de permettre d'anticiper le signalement des ruptures en temps réel à l'ensemble des intervenants concernés et de générer des alertes en cas de dysfonctionnements ou de fonctionnements anormaux des flux. Le but est de garantir que le Bon patient reçoive le Bon médicament au Bon moment, à la Bonne dose, selon la Bonne voie d'administration.

\section{Bibliographie}

Alavi M., Leidner D. E. (2001), Review: Knowledge Management and Knowledge Management Systems: Conceptual Foundations and Research Issues, Management Information Systems Quarterly, 25(1), 107-136.

Artiba A., Briquet M., Colin J. et al. (2004), Modélisation d'établissement de santé, $2^{e}$ conférence francophone en Gestion et Ingénierie de Systèmes Hospitaliers (GISEH).

Basole R. C., Bellamy M. A. (2013), Network Analysis of Supply Chain Systems: A Systematic Review and Future Research, Systems Engineering, (16), 235-249.

Bates D. W., Cullen D. J., Laird N. et al. (1995), Incidence of adverse drugevents and potential adverse drug events, JAMA, 274(1), 29-34. 
Bedouch P., Baudrant M. \& Detavernier M. (2009), La sécurisation du circuit du médicament dans les établissements de santé : données actuelles et expérience du centre hospitalier universitaire de Grenoble, Annales Pharmaceutiques Françaises, (67), 3-15.

Bernheim C., Schmitt E. \& Dufay E. (2005), Iatrogénie médicamenteuse nosocomiale et gestion des risques d'erreur médicamenteuse : à propos de l'analyse des notifications du réseau REEM, Oncologie, (7), 104-119.

Bouchand F., Thomas A., Zerhouni L. et al. (2007), Interventions pharmaceutiques avant et après informatisation de la prescription dans un service de médecine interne, La Presse Médicale, 36(3), 410-418.

Bourget S., Allenet B., Bedouch P. et al. (2007), Service pharmaceutique en service de soins : quelle adéquation avec les attentes des prescripteurs ?, Journal de Pharmacie de Belgique, 62(4), 101-105.

Chabrol M., Féniès P., Gourgand M. et al. (2006), Un environnement de modélisation pour le système d'information de la supply chain : application sur le nouvel hôpital d'Estaing, Revue des Sciences et Technologies de l'Information - Série ISI : Ingénierie des Systèmes d'Information, Lavoisier, 11(1), 137-162.

Ducret E. (2016), Management du risque à l'hôpital : Méthode AMDEC et actions de sécurisation appliquées à l'activité de nutrition parentérale pédiatrique au CHU de Nantes, Thèse de doctorat, Université d'Angers.

Ellenberg E., De Paris (2003), Management des risques à l'hôpital, Revue ADSP, 45, 63-66.

Evrard-Samuel K., Ruel S. (2013), Systèmes d'information et résilience des chaînes logistiques globales, Systèmes d'information \& management, 18 (1), 57-85.

Fabbe-Costes N. (1997), Les multiples rôles du système d'information et de communication dans l'action stratégique, in Marie-José Avenier (dir.), La stratégie “Chemin Faisant”, Economica, 189-215.

Jüttner U., Peck H. \& Christopher M. (2003), Supply chain risk management: outlining an agenda for future research, International Journal of Logistics: Research and Applications, 6(4), 197-210.

King W.J., Paice N., Rangrej J. et al. (2003), The effect of computerized physician order entry on medication errors and adverse drug events in pediatric inpatients, Pediatrics, 112, 506-509.

Koppel R., Metlay J. P., Cohen A. et al. (2005), Role of computerized physician order entry systems in facilitating medication errors, Journal of American Medical Association, 293(10), 1197-1203.

Lamarque F. (2014), Directeurs Supply Chain, tirez-vous le meilleur de votre (D)SI ?, Supply Chain Magazine, (86), 84-85.

Lavastre O., Gunasekaran A. \& Spalanzani A. (2014), Effect of firm characteristics, supplier relationships and techniques used on supply chain risk management (SCRM): An empirical investigation on french indus- 
trial firms, International Journal of Production Research, 52(11), 33813403.

Livolsi L., Fabbe-Costes N. (2004), La centralité des systèmes d'information (S.I) dans la fonction logistique, Revue française de Gestion Industrielle, 23(4), 27-44.

Ponomarov S., Holcomb M. (2009), Understanding the concept of supply chain Resilience, The International Journal of Logistics Management, 20(1), 124-143.

Reix R., Fallery B., Kalika M. et al. (2011), Systèmes d'information et management des organisations, Vuibert, Paris.

Ruel S., Da Silva C. F. (2016), Non-alignement stratégique des Systèmes d'Information et vulnérabilité de la Supply Chain : proposition d'un modèle, Logistique \& Management, 24(3-4), 179-185.

Said S. (2006), Le système d'information : système nerveux des supply chains, de l'intégration à la modularisation, Réalité industrielle, (56), 47-55.

Schmitt D., Allenet B., Brudieu E. et al. (2001), Acceptabilité de la prescription informatisée à l'hôpital. Enquête auprès de 44 médecins prescripteurs, Presse Medicale, (30), 1478-1481.

Vialle V., Tiphine T., Poirier Y. et al. (2011), Connaître, comprendre et lutter contre les erreurs médicamenteuses induites par l'informatisation du circuit du médicament, Annales Pharmaceutiques Françaises, 69, 165176.

Yin R.K. (2013), Case Study Research: Design and Methods. London : Sage Publications. 